\title{
Ultrasonic measurement of the reaction kinetics of the setting of calcium sulfate cements using implicit calibration
}

\author{
Johan E. Carlson ${ }^{a *}$ and Veli-Matti Taavitsainen ${ }^{\mathrm{b}}$
}

\begin{abstract}
Injectable bone cements based on calcium sulfate and calcium phosphates are being developed for use as bone defect filling and reinforcement of osteoporotic bones. For developers and end-users, kinetic properties of the setting reaction of such cements are of great interest. Existing standards for setting time measurement are based on visual examination of the cement surface and thus suffer from poor repeatability and subjectivity. Implicit calibration provides the means of determining parameters of a physical model at the same time as a calibration based on regression. This enables the use of indirect observations for the determination of implicit model parameters. In this paper, we study the hydration of calcium sulfate hemihydrate into calcium sulfate dihydrate, by combining multivariate calibration with a physical model of the reaction kinetics. The physical model contains three parameters, the reaction rate, the reaction order and a time delay. These parameters are estimated from ultrasound amplitude spectra. The resulting model fit has an $R^{2}$ value of $99.9 \%$. Copyright $\odot 2008$ John Wiley \& Sons, Ltd.
\end{abstract}

KEYWORDS: ceramic bone cements; ultrasound; reaction kinetics; implicit calibration; PLS regression

\section{INTRODUCTION}

Injectable bone cements [1] based on calcium sulfate and calcium phosphates are potential materials for bone defect filling and reinforcement of osteoporotic bone. They are especially suitable for filling fractured vertebras thanks to their good injectability even through small needles. The cements are biodegradable and will be replaced by new bone tissue over time.

There are a number of material properties of interest to both producers and medical personnel, since these properties have implications on the usability of the cements. For example, the porosity and mechanical strength of the final product is important since it affects the biodegradability and thus the ability for natural bone to grow into the structure and replace it.

Another very important property is the setting time, that is the time it takes for the cement to become hard enough for the wound to be closed without the risk of medical complications. More specifically, it is of interest to be able to characterize the entire setting reaction, since the applicability depends not only on the final setting time, but also on the workability of the cement during the initial period after mixing. It would also be of interest to researchers developing the cements to be able to model how the experimental conditions affect the reaction kinetics.

The existing standards for setting time measurement, the Vicat needle [2] (ASTM C191-92) and the Gillmore needles [3] (ASTM C266-89), are based on visual inspection of the cement surface subsequent to applying a standardized pressure to it. By the dependency on visual examination, these standards suffer from poor repeatability and subjectivity. Furthermore, the standards only provide information about the setting time, and no quantitative information about the progress of the setting reaction.
Other researchers have attempted to monitor the kinetics of the reaction in calcium phosphate cements, by modeling the rate of conversion in the reaction from intensity peaks in X-ray diffraction measurements $[4,5]$. The drawback of this approach is that samples need to be withdrawn during different stages of the reaction, and the reaction has to be stopped (by liquid nitrogen freezing). The samples are then prepared for X-ray diffraction measurements. This procedure is very time consuming and although it provides useful information about the kinetics of the reaction, it is not suitable for on-line use in any large-scale experimentation.

Since the mechanical properties of the cement change during the reaction, the use of ultrasound has potential, since this is in fact only a mechanical vibration and thus dependent on the material properties. Hence, by monitoring the evolution of some acoustic properties as a function of the setting time, it should be possible to follow the progress of the reaction. This idea was investigated by Carlson et al. [6] and Nilsson et al. [7] by monitoring the evolution of the acoustic impedance and the sound velocity using pulse-echo ultrasound. A similar approach based on studying the attenuation of ultrasound was proposed by Viano et al. [8]

\footnotetext{
* Department of Computer Science and Electrical Engineering, Luleå University of Technology, Lulea, Sweden.

E-mail:johan.carlson@ltu.se

a J. E. Carlson

Department of Computer Science and Electrical Engineering, Luleå University of Technology, Lulea, Sweden

b V.-M. Taavitsainen

Department of Marthemaitics, EVTEK University of Applied Sciences, Espoo, Finland
} 


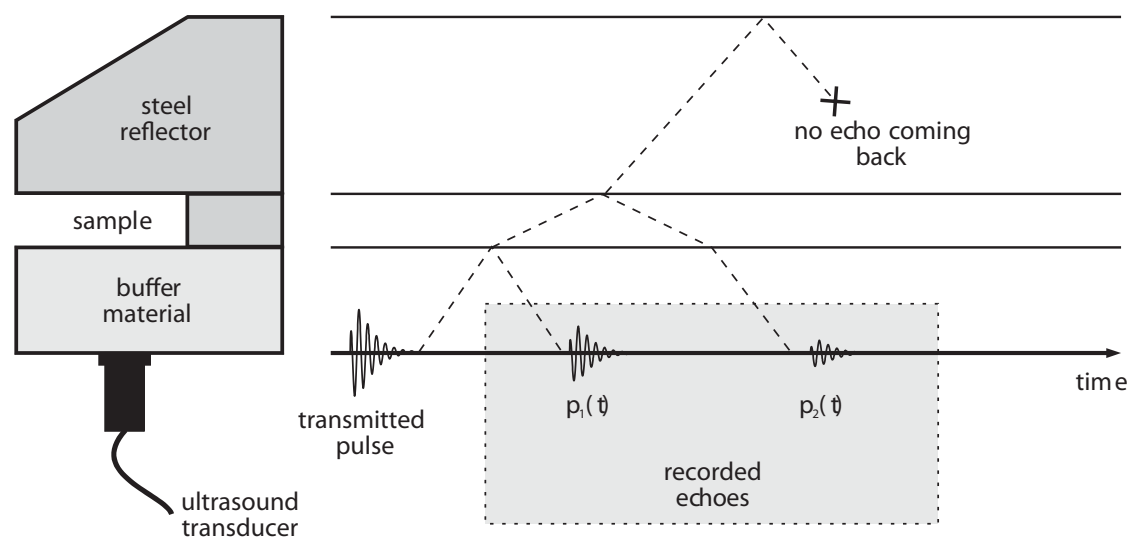

Figure 1. The pulse-echo reflectometer principle.

The ultrasonic techniques mentioned above share the benefit over the existing standards in the sense that they provide an objective means of on-line assessment of the progress of the reaction. However, no attempts were made in connecting the observed variations in acoustic properties to any underlying reaction mechanism. In this paper, we show how the principle of implicit calibration [9-11] can be used to combine measurement of ultrasound properties with an underlying model of the rate of the reaction. Other applications of fitting reaction models to multivariate data can be found in the papers by Knutson et al. [12] and Puxty et al. [13]. In addition to the ability to measure the setting time of the cement, these techniques also connect the observed variations in measured properties to the underlying physical mechanisms of the reaction.

The remainder of this paper is organized as follows. First, the principle of the ultrasonic measurement configuration is described, followed by a brief summary of the reaction kinetics of the setting of calcium sulfate cements. After this, the theory of direct and indirect implicit calibration is summarized, defining the notations used later in the paper. Section 3 describes the experimental setup followed by the experimental results. Sections 4 and 5 contain discussions about the results, followed by some concluding remarks.

\section{THEORY}

This section describes the theories of the measurement principle, the model of the reaction kinetics and the principles of direct and indirect implicit calibration.

\subsection{Ultrasound measurement principle}

The propagation of sound waves is a mechanical vibration and as such it depends on the mechanical properties of the material to be investigated. Non-destructive evaluation of materials using ultrasound is often performed in either through-transmission or pulse-echo mode. In the former, two ultrasound transducers are used, placed at the opposite side of the material sample, with one acting as a transmitter and the other as a receiver. This enables the measurement of attenuation and sound velocity relative to a calibration experiment (e.g. with only a water buffer between the transducers. The other approach is the pulse-echo principle, which is the one used in this work. Here, the transmission and reception of the sound waves are made with one single transducer. The transducer can be either in direct contact with the material or attached to a buffer which is then in contact with the material. In this work, we used the pulse-echo setup as described in Figure 1.

An ultrasound transducer transmits a short pulse, generally unknown. This is then reflected at the boundary between the buffer material and the sample, and at the boundary between the sample and the back reflector. These two echoes, denoted $p_{1}(t)$ and $p_{2}(t)$ are then recorded, and can then be used to calculate the attenuation and speed of sound through the sample material. If the acoustic properties of the buffer material are known, additional properties can be calculated such as acoustic impedance, adiabatic bulk modulus and density [14]. In this paper, we use the spectral amplitude of the first echo, denoted $p_{1}(t)$ in Figure 1. Throughout the setting reaction pulses were recorded and sampled using a digitizing oscilloscope with a sampling frequency of $200 \mathrm{MHz}$ and a resolution of 8 bits. The discrete versions of the pulses are denoted $p_{k}[m]$, where the subscripts $k=1,2, \ldots, N$ correspond to different times during the setting reaction. Their spectral representations, $P_{k}[q]$ were calculated using the discrete Fourier transform, that is

$$
P_{k}[q]=\sum_{m=1}^{M} p_{k}[m] e^{\frac{-j 2 \pi(q-1)(m-1)}{M}}
$$

where $M$ is the length of the sampled signal. The spectral amplitudes, $y_{k}[q]$ are then calculated as

$$
y_{k}[q]=\left|P_{k}[q]\right|=\sqrt{\Re\left\{P_{k}[q]\right\}^{2}+\Im\left\{P_{k}[q]\right\}^{2}}
$$

where $\Re\{\cdot\}$ and $\Im\{\cdot\}$ denote the real and imaginary parts, respectively. These were stored in the matrix $\mathbf{Y}$, defined as

$$
\mathbf{Y}=\left[\begin{array}{c}
\mathbf{y}_{1} \\
\mathbf{y}_{2} \\
\vdots \\
\mathbf{y}_{N}
\end{array}\right]
$$

where $\mathbf{y}_{k}=\left[y_{k}[1], y_{k}[2], \ldots, y_{k}[M]\right]$. In the following, the matrix $\mathbf{Y}$ is considered to be our observations, or measured data. 


\subsection{Reaction kinetics}

Calcium sulfate hemihydrate reacts with water, forming calcium sulfate dihydrate, according to the following reaction

$$
\mathrm{CaSO}_{4} \cdot \frac{1}{2} \mathrm{H}_{2} \mathrm{O}+\frac{3}{2} \mathrm{H}_{2} \mathrm{O} \rightarrow \mathrm{CaSO}_{4} \cdot 2 \mathrm{H}_{2} \mathrm{O}
$$

Several reaction mechanisms have been proposed for this reaction. The most common one is the semi-empirical Avrami equation. [15] A comparative study is given in Hand [15]; however, a generally approved mechanism does not exist. The reaction is assumed to have a nucleation period during which microscopic small dihydrate crystals are formed. After the nucleation period, the main reaction starts and it is either diffusion controlled or surface controlled depending on the supersaturation conditions of the mixture. It is generally approved that the apparent reaction order with respect to the mass fraction of hemihydrate depends on the controlling mechanism. According to these principles, the following kinetic mechanism is proposed [17]

$$
\left\{\begin{array}{l}
X_{A}^{\prime}=-k X_{A}^{p} \\
X_{B}^{\prime}=-\frac{3 M_{B}}{2 M_{A}} k X_{A}^{p} \\
X_{C}^{\prime}=k \frac{M_{C}}{M_{A}} X_{A}^{p}
\end{array}\right.
$$

where $A$ corresponds to calcium sulfate hemihydrate, $B$ stands for water and $C$ stands for calcium sulfate dihydrate. The corresponding mass fractions are denoted by $X_{A}, X_{B}$ and $X_{C}$, and their molecular weights are denoted by $M_{A}, M_{B}$ and $M_{C}$. The apparent reaction order is denoted by $p$ and the reaction rate by $k$. In addition to this, the main reaction is assumed to start after the nucleation period $t_{0}$. The model contains three unknown parameters $-k, p$ and $t_{0}$-to be estimated from the ultrasonic measurements using implicit calibration.

In addition to the model described in Equation (4), additional models were also tested, such as ordinary second-order kinetics, fractional-order kinetics using unknown exponents and Avrami kinetics, commonly used for describing crystal growth. However, the mechanism proposed here showed the best fit to the data.

\subsection{Implicit calibration}

In implicit calibration, the kinetic parameters are estimated simultaneously with the parameters of the calibration model which links the measured ultrasonic amplitude spectra to the mass fractions of the three compounds. The implicit calibration can be made in two different ways, in the indirect and in the direct way. In the former, the measured amplitude spectra at different times are calibrated to match the modeled mass fractions using some multivariate calibration technique, typically PLS regression [16]. In the latter, the modeled mass fractions are calibrated to match the measured amplitude spectra at different times, typically using ridge regression with non-negativity constraints. In order to clarify the method, we shall use the notation that follows.

Let $\mathbf{Y}$ be the matrix of measured amplitude spectra where the $i$ th row contains the spectrum measured at the ith measurement time. Let $\mathbf{X}_{\theta}$ be the matrix of modeled mass fractions, where $\boldsymbol{\theta}$ denotes the vector of the unknown parameters and the ith row contains the modeled mass fractions at the ith measurement time. Now the direct implicit calibration scheme can be expressed as the following regression model

$$
\mathbf{Y}=\mathbf{X}_{\theta} \mathbf{B}_{\theta}+\mathbf{E}=\widehat{\mathbf{Y}}_{\theta}+\mathbf{E}
$$

where $\mathbf{B}_{\theta}$ represents the pure component amplitude spectra, known to be non-negative and $\mathbf{E}$ represents a matrix of residual errors. Ridge regression is needed since, due to stoichiometry, $\mathbf{X}_{\boldsymbol{\theta}}$ is not of full rank.

The indirect implicit calibration scheme can be expressed as the following regression model

$$
\mathbf{X}_{\theta}=\mathbf{Y B}_{\theta}+\mathbf{E}=\widehat{\mathbf{X}}_{\theta}+\mathbf{E}
$$

where $\mathbf{B}_{\theta}$ represents the matrix of the regression coefficients and $\mathbf{E}$ represents a matrix of residual errors. Note that $\mathbf{B}_{\theta}$ is not the same matrix in the alternative calibration schemes. PLS regression is a good choice because the spectra may contain variation that is not related to the changes in the mass fractions.

The simple idea behind implicit calibration is that the unknown matrix $\mathbf{B}_{\theta}$ is estimated simultaneously with $\boldsymbol{\theta}$ minimizing the sum of squares norm of $\mathbf{E}$ or some other measure for the goodness of fit. Actually, for reasons explained in Taavitsainen et al. [10], in indirect implicit calibration, the maximization of the coefficient of determination $R^{2}$ between $\widehat{\mathbf{X}}_{\theta}=\mathbf{Y} \mathbf{B}_{\theta}$ and $\mathbf{X}_{\theta}$ is a better choice.

Because estimation of $\boldsymbol{\theta}$ is a nonlinear problem and estimation of $\mathbf{B}_{\boldsymbol{\theta}}$ is a linear problem, assuming constant $\boldsymbol{\theta}$, efficient optimization requires separation of the linear and nonlinear steps.

\subsubsection{Algorithm 1 (direct implicit calibration)}

1. Make an initial guess for $\boldsymbol{\theta}$.

2. Using the present value of $\boldsymbol{\theta}$, solve the system of differential Equations (4) which gives the matrix $\mathbf{X}_{\theta}$ in Equation (5).

3. Estimate the matrix $\mathbf{B}_{\theta}$ using ridge regression with nonnegativity constraints and calculate $\widehat{\mathbf{Y}}_{\theta}=\mathbf{X}_{\theta} \mathbf{B}_{\theta}$ and $\mathbf{E}=$ $\mathbf{Y}-\widehat{\mathbf{Y}}_{\theta}$. The geometric mean of the $R^{2}$ values of each of the three columns of $\mathbf{E}$ is then calculated, which is the objective function of the optimization algorithm.

4. Calculate a new value for $\boldsymbol{\theta}$ using the chosen optimization algorithm, for example the simplex algorithm or the some gradient-based method.

5. Repeat steps $2-4$ until the chosen convergence criteria are met.

\subsubsection{Algorithm 2 (indirect implicit calibration)}

1. Make an initial guess for $\boldsymbol{\theta}$.

2. Using the present value of $\boldsymbol{\theta}$, solve the system of differential equations (4) which gives the matrix $\mathbf{X}_{\theta}$ in Equation (6).

3. Estimate the matrix $\mathbf{B}_{\theta}$ (in Equation (6)) using PLS regression and calculate $\widehat{\mathbf{X}}_{\theta}=\mathbf{Y B}_{\theta}$ and $R^{2}$ between $\mathbf{X}$ and $\widehat{\mathbf{X}}_{\theta}$. This is then the objective function of the optimization algorithm.

4. Calculate a new value for $\boldsymbol{\theta}$ using the chosen maximization algorithm, for example the simplex algorithm or the some gradient-based method.

5. Repeat steps 2-4 until the chosen convergence criteria are met.

\section{EXPERIMENTS}

In the following subsections, we describe the materials used and how the data acquisition was done. 


\subsection{Materials and methods}

Thirty grams $\alpha$-calcium sulfate hemihydrate (CSH) powder (Bo Ehrlander AB, Gothenburg, Sweden) was mixed with an aqueous solution of $2.5 \%$ (by weight of $\mathrm{Na}_{2} \mathrm{HPO}_{4}$ at a liquid-to-powder (L/P) ratio of $0.32 \mathrm{ml} \mathrm{g}^{-1}$, during $1 \mathrm{~min}$ to form a paste. During the setting, CSH hydrates into calcium sulfate demihydrate (CSD) following the reaction from Subsection 2.2. The mixing time was $1 \mathrm{~min}$, after which the paste was considered homogeneous. Thereafter, it was put inside the measurement cell (Figure 1) and formed so that it completely covered the area of the transducer surface. The ultrasonic measurement was started 3 min after the initial mixing started. The data acquisition then continued for $2 \mathrm{~h}$, with one pulse-echo measurement every $20 \mathrm{~s}$. All measurements were performed in a climate chamber at $37 \pm 1^{\circ} \mathrm{C}$.

The pulse-echo measurements were made using a $2 \mathrm{MHz}$ ultrasound transducer from Ceram AB (Lund, Sweden). The transducer was excited using a Panametrics P5800 pulser/receiver. The received pulse-echoes were sampled at $200 \mathrm{MHz}$ using a Nicolet 460 digitizing oscilloscope with a vertical resolution of 8 bits. All further processing was done in MATLAB (The MathWorks, Inc.).

\subsection{Results}

The implicit calibrations were carried out using both the direct and indirect methods. In the indirect method, one has to choose the PLS dimension and the method of scaling for the measurement data $(\mathbf{Y})$. In this work, the columns of $\mathbf{Y}$ were mean centered, but otherwise not scaled. Theoretically, because the matrix $\mathbf{X}_{\theta}$ is of rank one, due to the stoichiometry, only one PLS dimension should be needed.

First, we estimated all three unknown parameters, $k, t_{0}$ and $p$ simultaneously. All optimizations were carried out using the Nelder-Mead simplex algorithm. Both methods gave a value of the apparent reaction order, $p \approx 2.3$ suggesting, according to for example Hand [15] and Niemann [17], a surface controlled reaction. Since the fit was practically as good with the value $p=2$, we fixed the apparent reaction order to this value and re-estimated the other two parameters. The benefit of having only two values is that the goodness of fit can be described by a contour plot of either residual sum of squares (direct method) or of $R^{2}$ values (indirect method).

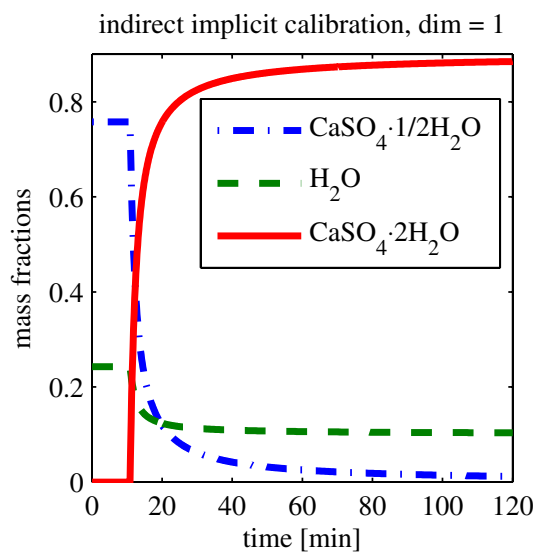

Table I. Estimated parameters using direct and indirect implicit calibration, respectively

\begin{tabular}{lcccccc|} 
Method & $k$ & $t_{0}$ & SS & $R^{2}$ & SE $(k)$ & SE $\left(t_{0}\right)$ \\
\hline Direct & 0.777 & 10.9 & 0.0155 & - & 0.021 & 0.067 \\
Indirect (dim = 1) & 0.778 & 10.9 & - & $99.89 \%$ & 0.027 & 0.079 \\
Indirect (dim = 2) & 0.826 & 11.1 & - & $99.94 \%$ & - & - \\
Indirect (dim = 3) & 0.831 & 11.1 & - & $99.94 \%$ & - & - \\
Indirect (dim = 4) & 0.849 & 11.1 & - & $99.97 \%$ & - & - \\
Indirect (dim = 5) & 0.864 & 11.1 & - & $99.97 \%$ & 0.111 & 0.833 \\
\end{tabular}

Using only one PLS dimension in the indirect implicit calibration gave practically the same results as the direct method. Using two PLS dimensions improved the optimal $R^{2}$ value slightly, but giving somewhat larger $k$ than the direct method. The changes both in the $R^{2}$ value and in parameter values were very small with higher dimensions than 2 (see Table 1). It can thus be said that, in this application, the indirect method is quite insensitive to the PLS dimension.

Table 1 shows the estimated parameters and the optimal residual sum of squares in the spectra (direct method) or the optimal geometric average of the $R^{2}$ values for the mass fractions (indirect method). The table also shows estimated standard errors (SE) for the parameters. Since only one measurement series was available, these estimates were obtained using a Jackknife principle [18]. The SE estimates were obtained by randomly selecting $2 / 3$ of the data and using these for the parameter estimation, repeating this procedure 200 times.

Since the mass fraction curves, estimated using both direct and indirect implicit calibration, are indistinguishable, only the curves obtained by the indirect method, with PLS dimensions 1 and 5 are shown in Figure 2. The differences between the two dimensions are almost indistinguishable by eye, though the parameter values are different (see Table 1).

The uniqueness of the two estimated parameters can be described by contour plots of the measured of goodness of fit (SS or $R^{2}$ ). Again, the $R^{2}$ contour lines of the indirect implicit calibration and SS contour lines of the direct implicit calibration are almost identical in form. Therefore, only $R^{2}$ contour lines of the indirect implicit calibration with PLS dimensions 1 and 5 are shown in Figure 3.

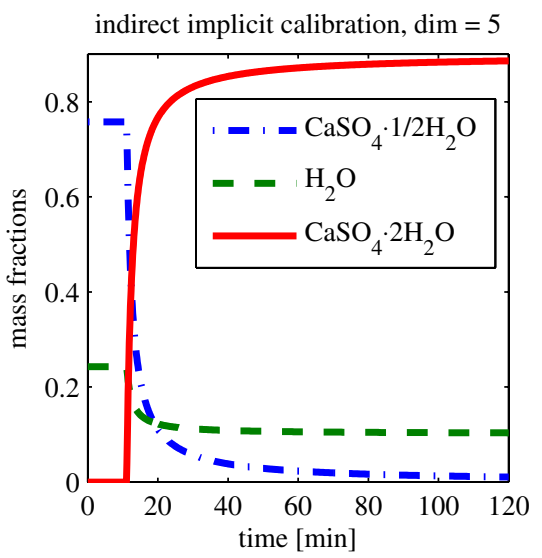

Figure 2. Mass fractions of the compounds of the reaction for indirect implicit calibration using one and five PLS components, respectively. This Figure is available in color online at www.interscience.wiley.com/journal/cem 

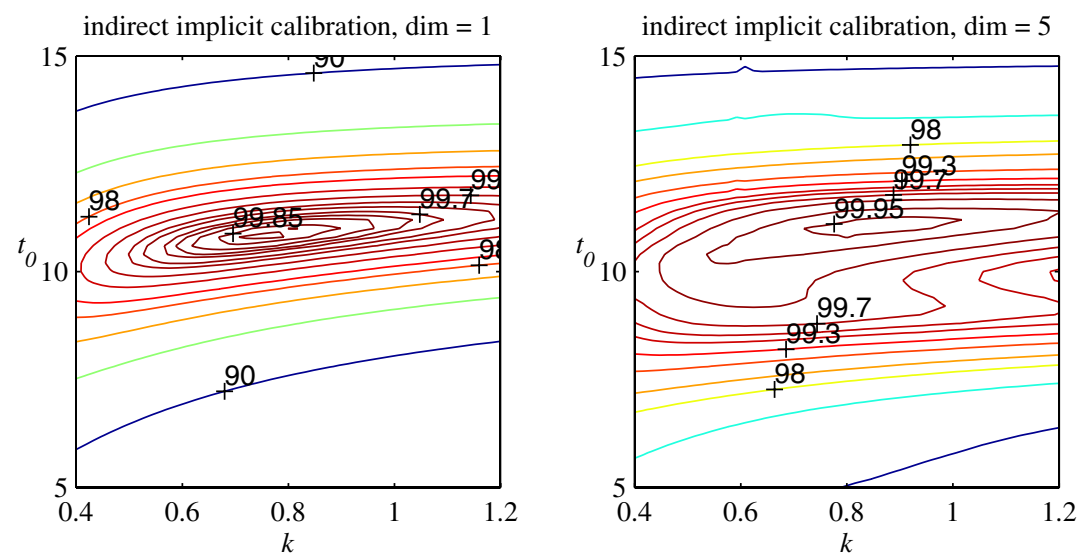

Figure 3. $R^{2}$ contour lines for indirect implicit calibration using one or five PLS components, respectively. This Figure is available in color online at www.interscience.wiley.com/journal/cem

Figure 3 also shows that too high a PLS dimension introduces some uniqueness problems. Direct implicit calibration allows the fit to be compared directly to the measured data. Figure 4 shows the measured spectra and the modeled spectra at chosen times. The fit is almost perfect, except during the first minute after the start of the main reaction. The reasons for this are discussed in Section 4.

\section{DISCUSSION}

In this paper, we have presented a method for monitoring the reaction kinetics of the setting of calcium sulfate-based cements. The work is based on the principle that properties of the ultrasound will change due to mechanical changes in the material. This idea was first exploited in the papers by Carlson et al. [6] and Nilsson et al. [7], but is here extended to incorporate knowledge about the underlying physics, that is the kinetics of the reaction. The main advantages from this are that:

- The observed data are projected onto a structure given by knowledge from the chemical reaction. As such, the measurement method should be more robust, since variations in data not related to the model are disregarded.

- The estimation of the kinetics model provides more insight on the underlying mechanisms of the reaction. This could prove useful to researchers in the field of material science. For endusers, such as medical personnel, this information is probably of less interest. However, the mass fraction of the end product (in this case CSD), is an indication of to which degree the reaction is set. As such, this method is a valuable tool also to the end-user.
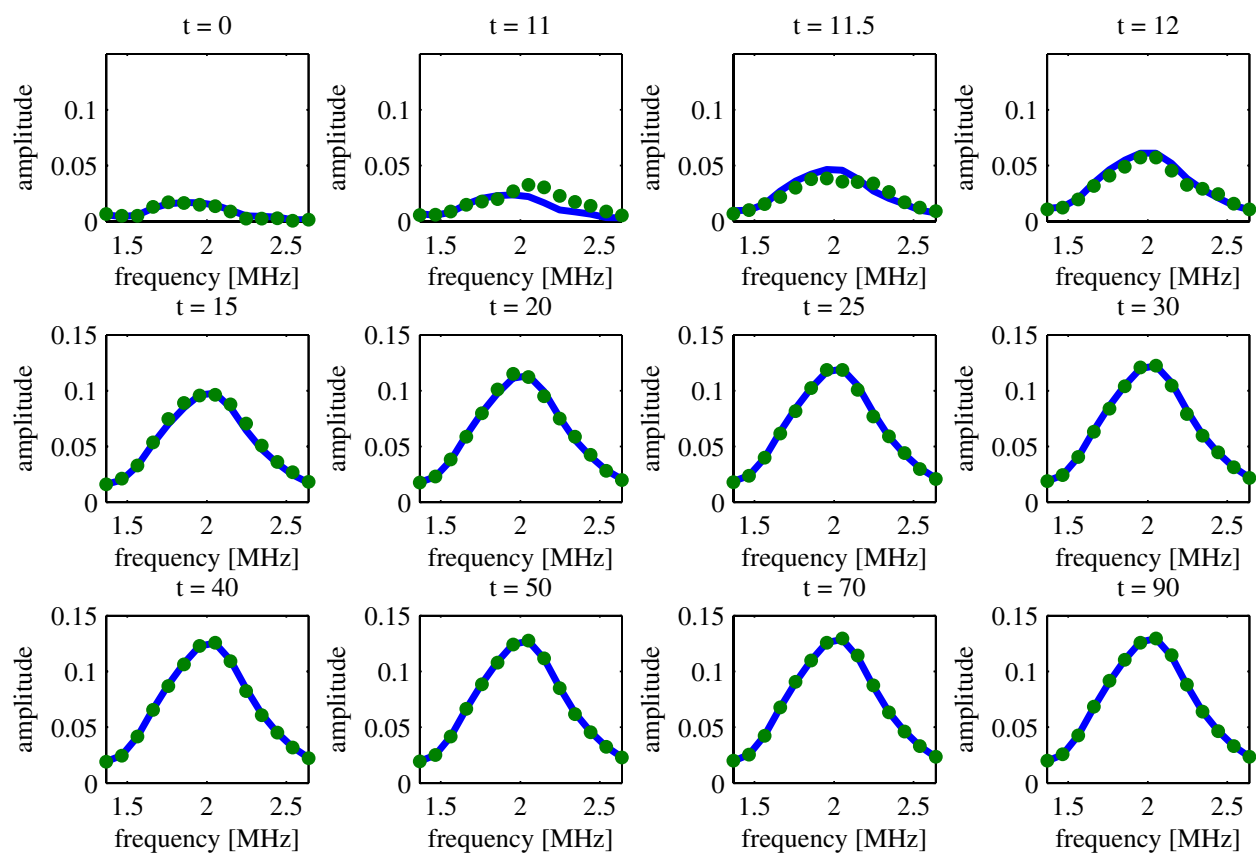

Figure 4. Measured and predicted ultrasound spectral amplitude spectra as a function of the reaction time, $t$, using direct implicit calibration. This Figure is available in color online at www.interscience.wiley.com/journal/cem 
In the previous work, the acoustic measurements were used to calculate some elementary mechanical properties of the material such as acoustic impedance, adiabatic bulk modulus and speed of sound $[6,7]$. The kinetics estimation presented in this paper could easily be combined with these results. This would then give further insight to the underlying mechanisms of the reaction.

The model in Equation (4) does not describe the reaction before $t=t_{0}$. In fact, we assume that the rate of change of the compounds is zero during this time period. This is obviously an over-simplification of the true mechanisms of the reaction, which also explains the poorer prediction results at the beginning of the reaction (see Figure 4). Until now, we have not been able to find any work that properly explains the governing reaction mechanism during this nucleation period. If the main goal of the measurements is, however, to estimate the setting time of the cement, this is not a serious limitation.

In this study, we chose to use only the spectral amplitude of the first pulse, reflected at the buffer-rod/cement interface (see Figure 1). There is actually more information available in the observed data than what we exploited here. During the setting reaction, not only is the CSH converting to CSD. There are also structural changes in the cement sample that will affect the ultrasound propagation. This information is, however, not useful in estimating the kinetics model, since the model does not attempt to describe this. Future research will focus on how to make use of additional information in the ultrasound data.

Other future research will be on other types of reactions, with other cement mixtures, more relevant for clinical applications. The work presented here is the first results showing that the principle of implicit calibration can be used in combination with ultrasound measurements for this type of problems.

\section{CONCLUSIONS}

In this paper, we presented a method based on ultrasound measurement and implicit calibration, for monitoring the setting of calcium sulfate cements. The methods combines a hard physical model, describing the reaction kinetics, with a soft (calibration) model linking variations in ultrasound attenuation to the progress of the reaction. The combination enables robust estimation of important properties of the reaction, rather than just studying changes in the acoustic properties.

We have showed that both indirect and direct implicit calibration give similar results, which suggests that the method is very robust.

The experimental results show an excellent model fit to the observed data, providing valuable information about the mechanisms of the reaction to researchers developing these materials.

\section{Acknowledgements}

The authors wish to express their sincerest gratitude toward $\mathrm{Dr}$ Malin Nilsson at BoneSupport AB for her valuable comments. The valuable comments from the reviewers, aiding in improving the manuscript, are also gratefully acknowledged.

\section{REFERENCES}

1. Nilsson M, Fernández E, Sarda S, Lidgren L, Planell JA. Microstructure analysis of novel resorbable calcium phosphate/sulphate bone cements. Bioceramics 2002; 14: 365-368.

2. Standard Test Method for Time of Setting of Hydraulic Cement by Vicat Needle. ASTM C191-92. Annual Book of ASTM Standards 1993; 04-01: 158-60.

3. Standard Test Method for Time of Setting of Hydraulic Cement Paste by Gillmore Needles. ASTM C266-89. Annual Book of ASTM Standards 1993; 04-01: 189-91.

4. Fernández E, Ginebra MP, Boltong FCM, Driessens FCM, Ginebra J, De Maeyer EAP, Verbeeck RMH, Planell JA. Kinetic study of the setting reaction of a calcium phosphate bone cement. J. Biomed. Mater. Res. 1996; 32: 367-374.

5. Sarda S, Fernández E, Nilsson M, Balcells M, Planell JA. Kinetic study of citric acid influence on calcium phosphate bone cements as water reducing agent. J. Biomed. Mater. Res. 2002; 61: 653-659.

6. Carlson J, Nilsson M, Fernández E, Planell JA. An ultrasonic pulse-echo technique for monitoring the setting of $\mathrm{CaSO}_{4}$-based bone cement. Biomaterials 2003; 24: 71-77.

7. Nilsson M, Carlson J, Fernández E, Planell JA. Monitoring the setting of calcium-based bone cements using pulse-echo ultrasound. j. Mater. Sci. Mater. Med. 2002; 13: 1135-1141.

8. Viano AM, Auwarter JA, Rho JY, Hoffmeister BK. Ultrasonic characterization of the curing process of hydroxyapatite-modified bone cement. J. Biomed. Mater. Res. 2001; 56: 593-599.

9. Taavitsainen V-M, Haario H. Rapid estimation of chemical kinetics by implicit calibration. I. J. Chemometrics 2001; 15: 215-239.

10. Taavitsainen V-M, Haario H, Laine M. Rapid estimation of chemical kinetics by implicit calibration. II. J. Chemometrics 2003; 17: 140-150.

11. Taavitsainen V-M, Jalava, J-P. Soft and harder multivariate modelling in developing the properties of titanium dioxide pigments. Chemometr. Intell. Lab. Syst. 1995; 29: 307-319.

12. Knutson J-R, Beechem J-M, Brand L. Simultaneous analysis of multiple fluorescense decay curves: a global approach. Chem. Phys. Lett. 1983; 102: 501-507.

13. Puxty $G$, Maeder M, Hungerbüller K. Tutorial on the fitting of kinetics model to multivariate spectroscopic measurements with non-linear least-squares regression. Chemometr. Intell. Lab. Syst. 2006; 81: 149164.

14. Kinsler LE, Frey AR, Coppens AB, Sanders J. Fundamentals of Acoustics (3rd edn). Wiley: New York, USA, 1982.

15. Hand RJ. The kinetics of hydration of calcium sulphate hemihydate: a critical comparison of the models in the literature. Cement Concr Res 1994; 24: 885-895.

16. Wold S, Sjöström M, Eriksson L. PLS-regression: a basic tool for chemometrics. Chemometr. Intell. Lab. Syst. 2001; 58: 109-130.

17. Niemann L. Die Reaktionskinetik des Gipsabbindens: Makroskopische Reaktionsraten und Mechanismen in molekularem Maßstab. Karlsruher Mineralogische und Geochemische Hefte 2005; 28: Universitätsverlag Karlsruhe.

18. Efron B, Tibshirani RJ. An Introduction to the Bootstrap. Chapman \& Hall: New York, USA, 1993. 